\title{
Resource Allocation for D2D Communication in Cellular Networks Based on Stochastic Geometry and Graph-coloring Theory
}

\author{
Fangmin $\mathrm{Xu}^{1 *}$, Pengkai Zou ${ }^{1}$, Haiquan Wang ${ }^{1}$, Haiyan $\mathrm{Cao}^{1}$, Xin Fang ${ }^{1}$, and Zhirui $\mathrm{Hu}^{1}$ \\ ${ }^{1}$ Institute of Communications Engineering, Hangzhou Dianzi University \\ Hangzhou, Zhejiang, China \\ [e-mail: fangminxu@gmail.com,645881071@qq.com,tx_wang@hdu.edu.cn, caohy@hdu.edu.cn, \\ feliciafang@hdu.edu.cn,huzhirui@hdu.edu.cn] \\ *Corresponding author: Fangmin $\mathrm{Xu}$
}

Received August 29, 2020; revised October 29, 2020; accepted December 7, 2020; published December 31, 2020

\begin{abstract}
In a device-to-device (D2D) underlaid cellular network, there exist two types of co-channel interference. One type is inter-layer interference caused by spectrum reuse between D2D transmitters and cellular users (CUEs). Another type is intra-layer interference caused by spectrum sharing among D2D pairs. To mitigate the inter-layer interference, we first derive the interference limited area (ILA) to protect the coverage probability of cellular users by modeling D2D users' location as a Poisson point process, where a D2D transmitter is allowed to reuse the spectrum of the CUE only if the D2D transmitter is outside the ILA of the CUE. To coordinate the intra-layer interference, the spectrum sharing criterion of D2D pairs is derived based on the (signal-to-interference ratio) SIR requirement of D2D communication. Based on this criterion, D2D pairs are allowed to share the spectrum when one D2D pair is far from another sufficiently. Furthermore, to maximize the energy efficiency of the system, a resource allocation scheme is proposed according to weighted graph coloring theory and the proposed ILA restriction. Simulation results show that our proposed scheme provides significant performance gains over the conventional scheme and the random allocation scheme.
\end{abstract}

Keywords: Device-to-Device (D2D), Spectrum Reuse, Interference, Stochastic Geometry, Weighted Graph-Coloring 


\section{Introduction}

Growing demands for providing high data rate and low energy cost to support the rapidly increased mobile data traffic have been a great impetus to the development of novel technologies of improving system capacity [1-6]. Device-to-device (D2D) communication has drawn much attention due to its short mutual distance and high spectrum reuse gain by allowing direct communications between two or more user equipment in proximity [2]. As a result, D2D has been considered in the fifth generation $(5 \mathrm{G})$ as an underlay to cellular networks [1].

On the other hand, the energy efficiency (EE) of the system is becoming mainstream pursuit with the growth of energy demand and the rise of energy prices [4]. A D2D network underlaying a cellular network can improve the spectrum efficiency (SE) and energy efficiency (EE) of the system [5-6], and reduce the traffic load of the macro BS [3]. However, resource sharing among cellular users and D2D users will cause serious co-channel interference.

To solve this problem, there has been extensive research on design and analysis of resource allocation schemes for D2D underlaid cellular networks [7-16]. Authors in [7] derived the average coverage probability of cellular users with corresponding density of potential D2D users based on stochastic geometry theory. In [8], a distance-based power control scheme was devised to enhance SE and coverage probability of users. The authors in [9] proposed a resource allocation and power control algorithm by exploiting the properties of fractional programming and penalty function to maximize the EE of DUEs, while the number of DUEs is less than that of CUEs. A two-loop iterative algorithm was designed in [10] to solve the energy efficiency fairness problem with the constraint of the maximum transmit power and the minimum data rate requirement. Yang et al. [11] investigated the energy-efficient power control problem for DUEs to maximize the total EE and the individual EE. The authors in [12] proposed a resource allocation algorithm which aimed to maximize the minimum weighted energy efficiency of DUEs as well as satisfy the minimum data rates of CUEs. In [13], a reverse iterative combinatorial auction (ICA) game was formulated to improve the system EE, while the QoS requirements of users were ignored. In [14], the authors formulated two resource allocation problems to optimize system EE and total individual EE considering the QoS of CUEs. An energy-efficient scheduling scheme among DUEs was proposed in [15], while only the QoS of DUEs was considered. A user grouping and power control scheme were proposed in [16] to maximize the system EE, while it only considered the impact of interference on DUEs when grouping, which may cause serious interference to CUEs.

Apart from these high complexity optimizing methods, the interference limited method was considered in [17-18] to reduce the computational complexity. A $\delta_{D}$-interference limited area (ILA) scheme was designed to reduce the interference from CUEs to D2D users in [17]. The authors in [18] proposed a minimum distance restriction to alleviate the interference from cellular users to D2D links reusing the same resources. However, these are generally designed to improve SE. Different from the exiting work, we consider the EE programming problem based on the QoS constraints of both CUEs and DUEs based on stochastic geometry modeling and weighted graph coloring to mitigate co-channel interference in D2D underlaid cellular networks.

In this paper, a resource allocation scheme in D2D underlaid cellular networks was proposed, where D2D transmitters reuse the uplink spectrum of CUEs. Resource reusing can improve the energy efficiency of the system but will cause serious interference to CUEs. To guarantee the QoS of CUEs, an interference limited area (ILA), which ensure that the SINR of the CUE 
be greater than a specified threshold with a large probability, is derived for CUEs based on the stochastic analysis. That is to say, the DUE who is out of ILA of the CUE is allowed to reuse the CUE's resource. Otherwise, resource sharing between the DUE and CUE is forbidden. Then, based on the above restrictions, we formulated a user grouping scheme that meet the DUE's distance restriction to avoid the DUEs suffer from serious interference. Finally, we propose an optimal resource selection scheme to maximize system EE based on the weighted graph-coloring theory. The main contributions in this paper are summarized as follows.

1) We propose a resource reuse scheme, which allows multiple D2D users reuse cellular user's spectrum resource, without increasing the overhead of conventional cellular system based on the stochastic geometry theory. And the energy efficiency of system can be greatly enhanced.

2) To depress the D2D-to-cellular interference, we propose an interference limited area for CUEs by modeling the D2D locations as a Poisson point process. Compare to the scheme in [16], the proposed scheme can efficiently protect the CUE's performance from serious interference.

3) In order to reduce the intra-layer interference among DUEs, we formulate a user grouping scheme based on the limitation of user distance. Based on the result of grouping scheme, we select an optimal DUE according to the interference graph to reuse the resource.

4) We demonstrate the effectiveness of our proposed resource reuse scheme by simulations.

The rest of the paper is organized as follows. In Section 2, we describe the system model and problem formulation to improve EE for D2D underlaid cellular networks. Section 3 describes the proposed resource allocation scheme based on ILA and the weighted graph coloring theory. The simulation results are given in Section 4. And we draw the conclusions in Section 5. Proof of the Theorem is given in the Appendix.

\section{System Model and Problem Formulation}

\subsection{System Model}

We consider an uplink scenario in a single-cell cellular system, in which there exist two kinds of communications, as illustrated in Fig. 1. One is called cellular communication. Another is D2D communication which is an underlay to the cellular communication. In the system, $M$ cellular user equipment (CUE) and $K$ D2D user equipment (DUE) $(M \leq K)$ are randomly distributed in the coverage area of the base station (BS). DUEs are distributed according to the Poisson point process (PPP) $\Phi$ of intensity $\lambda_{d}$. Each D2D pair consists of a D2D transmitter (DT) and a D2D receiver (DR) with a fixed distance $R_{D}$. There are $M$ orthogonal spectrum resources allocated to $M$ CUEs respectively. Without loss of generality, we assume that the $i$ th resource block is allocated to the $i$-th CUE. DUEs share the same resource set with CUEs. Therefore, the co-channel interference consists of three parts: 1) interference received by BS from DT; 2) interference received by DR from CUE; 3) interference received by DR from other DT sharing the same spectrum resource with that D2D pair. 


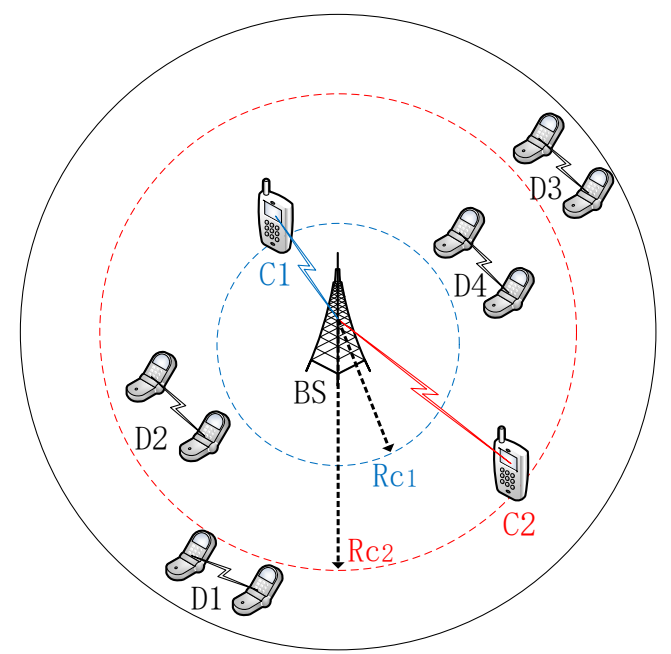

Fig. 1. System model and the illustration of resource allocation

In the following, we use $C_{i}(\mathrm{i}=1,2, \ldots, M)$ and $D_{j}(\mathrm{j}=1,2, \ldots, K)$ to denote the $i$-th CUE and $j$-th D2D pairs respectively; $D T_{j}$, and $D R_{j}$ represent the transmitter of $D_{j}$ and the receiver of $D_{j}$ respectively. The channel gain can be expressed as

$$
g=h \cdot \beta
$$

where $h$ represents the small scale fading, $h \sim \exp (\mu)$, and $\beta=d^{-\alpha}$ is the large scale fading, in which $d$ is the distance between transmitter and receiver; $\alpha$ is the path loss exponent. The useful symbol information is summarized as Table 1.

Table 1. The Implication of Symbols

\begin{tabular}{cl}
\hline \hline Symbols & Implication \\
\hline$g_{i}^{C}$ & channel gain between $C_{i}$ and BS \\
$g_{j}^{D}$ & channel gain between $D T_{j}$ and $\mathrm{BS}$ \\
$g_{i j}^{c d}$ & channel gain between $C_{i}$ and $D R_{j}$ \\
$g_{k j}^{d d}$ & channel gain between $D T_{k}$ and $D R_{j}$ \\
$d_{C_{i}, B}$ & distance from $C_{i}$ to BS \\
$d_{D_{j}, B}$ & distance from $D T_{j}$ to $\mathrm{BS}$ \\
$d_{C_{i}, D_{j}}$ & distance from $C_{i}$ to $D R_{j}$ \\
$d_{D_{k}, D_{j}}$ & distance from $D T_{k}$ to $D R_{j}$ \\
$\beta_{C_{i}, B}$ & large scale fading between $C_{i}$ and $\mathrm{BS}$ \\
$\beta_{D_{j}, B}$ & large scale fading between $D T_{j}$ and $\mathrm{BS}$ \\
$\beta_{C_{i}, D_{j}}$ & large scale fading between $C_{i}$ and $D R_{j}$ \\
$\beta_{D_{j}, D_{k}}$ & large scale fading between $D T_{j}$ and $D R_{k}$ \\
\hline \hline
\end{tabular}

The received Signal to Interference plus Noise Ratio (SINR) at the BS from $C_{i}$ and the SINR of $D_{j}$ using the $i$-th resource block can be represented as

$$
\operatorname{SINR}_{i}^{C}=\frac{P_{c} g_{i}^{C}}{\sum_{j=1}^{K} \pi_{i j} P_{d} g_{j}^{D}+N_{0}}
$$




$$
\operatorname{SINR}_{j}^{D(i)}=\frac{P_{d} g_{j j}^{d d}}{P_{c} g_{i j}^{c d}+\sum_{k \neq j}^{K} \pi_{i k} P_{d} g_{k j}^{d d}+N_{0}}
$$

where $P_{c}$, and $P_{d}$ is the transmit power of CUE and DT respectively; $N_{0}$ is the power of the additive white Gaussian noise; $\pi_{i j}$ is a resource reuse metrics. $\pi_{i j}=1$ indicates that $D_{j}$ shares the resources of $C_{i}$, while $\pi_{i j}=0$ indicates that $C_{i}$ and $D_{j}$ are not allowed to share the same resources. According to SINR in (2) and (3), we can write the energy efficiency (EE) of $C_{i}$ as

$$
E E_{i}^{C}=\frac{\log _{2}\left(1+\operatorname{SINR} R_{i}^{C}\right)}{P_{C}+P_{\text {cir }}}
$$

where $P_{c i r}$ represent the circuit power consumption of user. Similarly, the energy efficiency (EE) of $D_{j}$ can be expressed as

$$
E E_{j}^{D}=\frac{\log _{2}\left(1+\operatorname{SINR} R_{j}^{D}\right)}{P_{d}+2 P_{\text {cir }}}
$$

\subsection{Problem Formulation}

The EE of the system can be expressed as

$$
\begin{aligned}
E E_{\text {sum }} & =\sum_{i=1}^{M} E E_{i}^{C}+\sum_{j=1}^{K} E E_{j}^{D} \\
& =\sum_{i=1}^{M} \frac{\log _{2}\left(1+\operatorname{SINR} R_{i}^{C}\right)}{P_{c}+P_{\text {cir }}}+\sum_{i=1}^{M} \sum_{j=1}^{K} \pi_{i j} \frac{\log _{2}\left(1+\operatorname{SINR} R_{j}^{D(i)}\right)}{P_{d}+2 P_{\text {cir }}}
\end{aligned}
$$

We aim to maximize the system EE with the SINR constrain of CUEs and DUEs, i.e.,

$$
\begin{array}{ll} 
& \max . E E_{\text {sum }} \\
\text { s.t. } & \operatorname{SINR}_{i} \geq \operatorname{SINR}_{c} \quad \forall C_{i} \in \mathrm{C} \\
& \operatorname{SINR}_{j}^{D(i)} \geq \operatorname{SINR}_{d} \quad \forall D_{j} \in \mathrm{D} \\
& \sum_{i=1}^{M} \pi_{i j} \leq 1 \quad \forall \mathrm{j} \in\{1, \ldots, \mathrm{K}\}
\end{array}
$$

where constraints (7a) and (7b) guarantee the QoS of CUE and DUE respectively, and constraint (7c) indicates that $D_{j}$ is only allowed to reuse at most one cellular user's resource at each time. However, in the optimization problem (7), the number of spectrum allocation schemes for $K$ D2D pairs is as huge as $M^{K}$. Hence, we try to obtain a low complexity scheme based on the stochastic geometry and graph coloring.

\section{The Proposed Resource Allocation Scheme}

In this section, we proposed a two-step resource allocation scheme to mitigate the inter-layer interference between CUEs and DUEs and intra-layer interference among users. Firstly, we derive an ILA based on stochastic geometry theory, where only DUEs outside the ILA of the CUE are allowed to reuse the spectrum with the CUE. Then, based on the ILA, we propose a multi-user resource sharing solution according to the interference graph. 


\subsection{ILA of Cellular Users}

Since the cellular users are the primary users of cellular systems, we formulate an ILA to guarantee the QoS of the CUEs where DUEs outside the ILA are allowed to reuse the spectrum of the CUE.

The definition of the ILA can be thought of equivalently as the coverage probability of the CUEs interfered by a transmitter outside $A_{0}$ should be large enough [19]. Therefore, the ILA of CUEs can be derived by the coverage probability defined as $\mathrm{P}(\mathrm{SINR}>\mathrm{T})$. The design of ILA must, therefore, ensure that the SINR of the CUE be greater than a specified threshold with a large probability $\beta$, such that

$$
P\left(\operatorname{SINR}_{i}^{C}>T\right) \geq \beta
$$

Unlike the existing work where interference D2D pairs are placed deterministically on a regular grid, we model their location as a PPP process with intensity $\lambda_{d}$. Hence, the SINR in (2) can be rewritten as

$$
\operatorname{SINR}_{i}^{C}=\frac{P_{c} h_{i} d_{C_{i, B}}^{-\alpha}}{N_{0}+P_{d} h_{0} R_{0}^{-\theta}+\sum_{j \in \Phi \backslash A_{0}} P_{d} h_{j} d_{D_{j, B}}^{-\theta}}
$$

where $P_{c} h_{i} d_{C_{i}, B}^{-\alpha}$ and $P_{d} h_{0} R_{0}^{-\theta}$ are the received signal power and the interference power caused by the D2D users at a random distance $R_{0}$ with path loss exponent $\theta>2$, respectively. $\sum_{j \in \Phi \backslash A_{0}} P_{d} h_{j} d_{D_{j, B} B}^{-\theta}$ is the cumulative interference from D2D transmitters outside $A_{0} ; A_{0}$ is an interference limited area with $\mathrm{BS}$ as the center and $R_{0}$ as its radius.

Lemma 1: $\xi$ is a random variable with probability density function (p.d.f.) $f_{\xi}(\mathrm{x}), h \sim \exp (\mu), \tau$ is a positive constant. Then, we have

$$
P\left(\frac{h}{\xi}>\tau\right)=E_{\xi} e^{-\mu \tau \xi}
$$

Proof:

$$
\begin{aligned}
P\left(\frac{h}{\xi}>\tau\right) & =E_{\xi} P(h>\tau \xi) \\
= & E_{\xi} \int_{\tau \xi}^{\infty} \mu e^{-\mu x} d x \\
= & E_{\xi} e^{-\mu \tau \xi}
\end{aligned}
$$

Theorem 1: The ILA of a tagged CUE is an inner region of a circle with the BS as the center and $R_{0}$ as the radius, where

$$
R_{0} \geq f^{-1}(\beta)
$$

with

$$
f\left(R_{0}\right)=\frac{\exp \left(-\frac{\mu T d_{c}^{\alpha} N_{0}}{P_{c}}\right)}{1+\frac{T d_{c}^{\alpha} P_{d} R_{0}^{-\theta}}{P_{c}}} \exp \left(-\frac{\pi \lambda_{d}}{M}\left(\frac{T P_{d} d_{c}^{\alpha}}{P_{c}}\right)^{\frac{2}{\theta}} \int_{R_{0}^{2}\left(\frac{P_{c}}{T P_{d} d_{c}^{\alpha}}\right)^{\frac{2}{\theta}}}^{R_{C}}\left(\frac{1}{1+u^{\frac{\theta}{2}}}\right) d u\right)
$$

and $f^{-1}$ is the inverse function determined by $f$ uniquely; $R_{C}$ is the cell radius. Theorem 1 indicates that DUEs out of the ILA $A_{0}$ can share spectrum with the tagged CUE.The proof of 
theorem 1 were given at the appendix.

\subsection{Interference limited threshold of DUEs}

After implementing the above step, we consider the intra-layer interference among users who share the same spectrum resource. Therefore, we introduce a mutual interference restrictions mechanism based on the distance.

Similar to [17], the Signal-to-interference ratio (SIR) restriction of $D_{j}$ between other DUEs and CUEs can be expressed as follows

$$
\begin{gathered}
\operatorname{SIR}_{D_{j} \leftarrow D_{k}}=\frac{R_{D}^{-\theta}}{d_{D_{k}, D_{j}}^{-\theta}} \geq \mu_{d} \\
\operatorname{SIR}_{D_{j} \leftarrow C_{i}}=\frac{P_{d} R_{D}{ }^{-\theta}}{P_{C} d_{C_{i} D_{j}}^{-\theta}} \geq \mu_{d}
\end{gathered}
$$

where $\mu_{d}$ is the minimum SIR restriction of DUEs; $S I R_{D_{j} \leftarrow D_{k}}$ represent the SIR from $D_{k}$ to $D_{j}$, $S I R_{D_{j} \leftarrow C_{i}}$ represent the SIR from $C_{i}$ to $D_{j}$. Thus the distance restriction of $D_{j}$ between CUE and DUE can expressed as

$$
\begin{aligned}
& d_{D_{k}, D_{j}} \geq R_{D} \mu_{d}^{1 / \theta} \\
& d_{C_{i}, D_{j}} \geq R_{D}\left(\frac{P_{c}}{P_{d}} \mu_{d}\right)^{1 / \theta}
\end{aligned}
$$

\subsection{Optimal Resource Reuse Scheme Based on Graph-Coloring}

After the above two steps, we can obtain the set of DUEs who can reuse the resources of $C_{i}$, and it can be expressed as $\Phi_{i}=\left\{D_{j}, \ldots,(j \in 1,2, \ldots K)\right\}$; and the set of CUEs that can share the resources with $D_{j}$ can be expressed as $\Psi_{j}=\left\{C_{i}, \ldots,(i \in 1,2, \ldots M)\right\}$.

As depicted in Fig. 1, the protection area of cellular user $C_{1}$ and $C_{2}$ can be expressed as the circular area with the base station as their center, with $R_{C 1}$ using blue line and $R_{C 2}$ using red line as their radius, respectively. Considering the case that one DUE has more than one CUEs that can be chosen to share the spectrum. For example, $D_{1}$ is out of the ILAs of $C_{1}$ and $C_{2}$. To maximize EE of the system, we formulate a metric to choose the best resource based on weighted graph-coloring [20]. The EE of $C_{i}$ and $D_{j}$ can be expressed as

$$
E E=\frac{\log _{2}\left(1+\frac{\beta_{c_{i}, B}}{\beta_{D_{j}, B}}\right)}{P_{c}+P_{c i r}}+\frac{\log _{2}\left(1+\frac{\beta_{D_{j}, D_{j}}}{\beta_{c_{i}, D_{j}}}\right)}{P_{d}+2 P_{c i r}}=\log _{2}\left(\left(1+\frac{\beta c_{i, B},}{\beta_{D_{j}, B}}\right)^{\frac{1}{P_{d}+2 P_{c i r}}} \cdot\left(1+\frac{\beta_{D_{j}, D_{j}}}{\beta_{C_{i}, D_{j}}}\right)^{\frac{1}{P_{c}+P_{c i r}}}\right)
$$

From (14), the total EE of $C_{i}$ and $D_{j}$ depends on

$$
\eta_{C_{i}, D_{j}}=\left(1+\frac{\beta_{C_{i} B}}{\beta_{D_{j}, B}}\right)^{\frac{1}{P_{d}+2 P_{c i r}}} \cdot\left(1+\frac{\beta_{D_{j}, D_{j}}}{\beta_{c_{i}, D_{j}}}\right)^{\frac{1}{P_{C}+P_{c i r}}}
$$


If $P_{C}=P_{d}+P_{c i r}$, the total EE of $C_{i}$ and $D_{j}$ is an increasing function of $\eta_{C_{i}, D_{j}}$

$$
\eta_{C_{i}, D_{j}}=\frac{\beta_{C_{i}, B}}{\beta_{D_{j}, B}}+\frac{\beta_{D_{j}, D_{j}}}{\beta_{C_{i}, D_{j}}}
$$

Hence, we choose the CUE who has the maximal $\eta_{c_{i}, D_{j}}$ with $D_{j}$ to share the spectrum of $D_{j}$. Fig. 2 shows the interference between different type of users as follows:

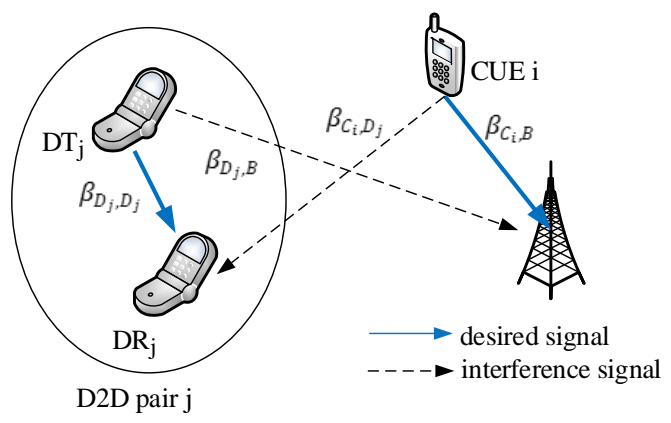

a. interference between CUE and DUE

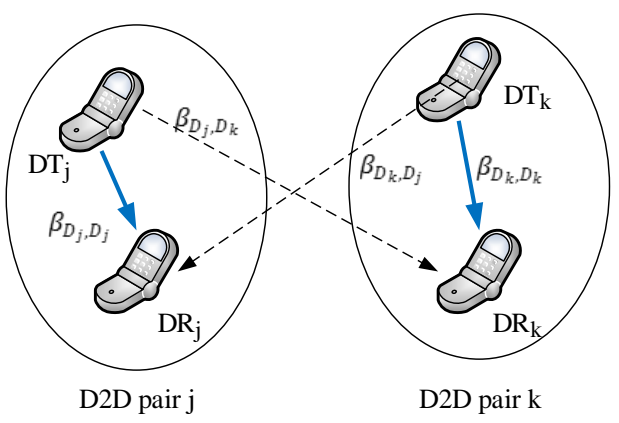

b. interference between D2D pairs

Fig. 2. Interference between different type of users

The proposed algorithm can be summarized as Algorithm 1 .

Algorithm 1 Proposed resource allocation algorithm

1) Initialize the location of CUEs and DUEs according to Poisson point process;

2) BS get user's location information and calculate the channel gain from (1);

3) Initialize the reusable candidate DUEs for $C_{i}: \Phi_{i}=\left\{D_{1}, D_{2}, \ldots, D_{K}\right\}$, candidate CUEs for $D_{j}: \Psi_{j}=$ $\emptyset$, the optimal reusable DUEs for $C_{i}: \Phi_{i}{ }^{*}=\varnothing$, the optimal reusable CUEs for $D_{j}: \Psi_{j}{ }^{*}=\varnothing$;

\section{Perform the resource allocation algorithm:}

for $i \in \mathrm{C}$

for $j \in \mathrm{D}$

$$
\text { if } d_{D_{j}, B}>R_{C_{i}} \text { and } S I R_{D_{j} \leftarrow C_{i}}>\mu_{d}
$$

for $\mathrm{k} \in \Phi_{i} \backslash j$

if $\operatorname{SIR}_{D_{j} \leftarrow D_{k}}>\mu_{d}$

$\Psi_{j}=\Psi_{j} \cup i$

endif

endfor

else

endfor

$\Phi_{i}=\Phi_{i} \bigvee$

endfor

for $j \in \mathrm{D}$

$i^{*}=\underset{i \in \Psi_{j}}{\operatorname{argmax}} \eta_{C_{i}, D_{j}}$, select a CUE to reuse which has the maximal $\eta$ with $D_{j}$

endfor

$\Psi_{j}{ }^{*}=i, \Phi_{i}{ }^{*}=\Phi_{i}{ }^{*} \cup j$, completed user grouping 


\section{Simulation Results and Analysis}

To validate the proposed algorithm, we compare the algorithm with conventional algorithm in [16] and random algorithm by simulation. In the random algorithm, it performs the user grouping scheme the same as the proposed scheme while select reused resource randomly. The rest parameters of our simulation are summarized in Table 2.

Table 2. Simulation Parameters

\begin{tabular}{ll}
\hline \hline Parameters & Values \\
\hline cell radius $/ R_{C}$ & $500 \mathrm{~m}$ \\
power of CUEs $/ P_{c}$ & $25 \mathrm{dBm}$ \\
power of DUEs $/ P_{d}$ & $20 \mathrm{dBm}$ \\
circuit power $/ P_{c i r}$ & $10 \mathrm{~mW}$ \\
D2D link distance $/ R_{D}$ & $20 \mathrm{~m}$ \\
noise power $\mathrm{N}_{0}$ & $-114 \mathrm{dBm}$ \\
number of CUEs & 10 \\
density of DUEs $/ \mathrm{km}^{2}$ & $20-100$ \\
path loss exponent $\alpha$ & 4 \\
path loss exponent $\theta$ & 3 \\
threshold $\gamma_{1}, \gamma_{2}[16]$ & $10 \mathrm{~dB}$ \\
SIR restriction of DUEs $/ \mu_{d}$ & $10 \mathrm{~dB}$ \\
simulation times & 1000 \\
\hline \hline
\end{tabular}

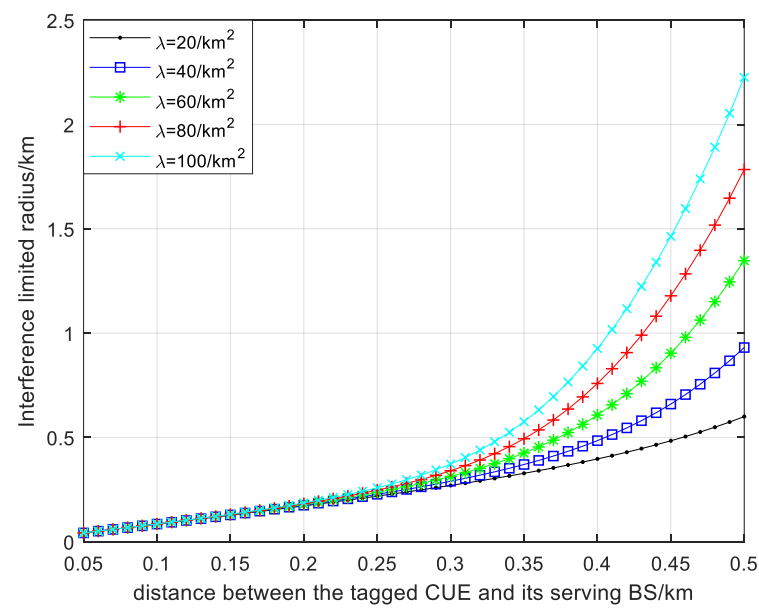

Fig. 3. Interference limited radius R0 v.s. $\lambda$ for different distance between the tagged CUE and its serving BS with $\beta=0.8$, and $\mathrm{T}=-4 \mathrm{~dB}$.

Fig. 3 describes the interference limited radius for different distance between the tagged CUE and its serving BS. Simulation results in Fig. 3 show that the ILA of CUEs is influenced by the distance between CUE and BS and density of DUEs. The higher the distance is, the larger ILA is, and the size of ILA increases with the increasing of density of DUEs as well. 


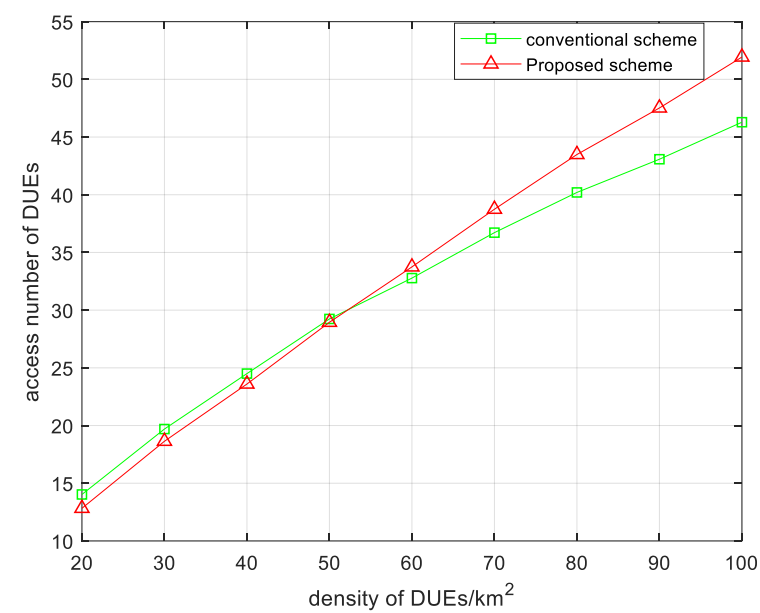

Fig. 4. Access number of DUEs for different density of DUEs

Fig. 4 gives the access number of DUEs for different density of DUEs. It shows that with the increasing of the total number of DUEs, more DUEs are allowed to access the system. As observed, the conventional scheme can access a little more users when the density of DUEs was low because it did not restrict for specific users. While when the density of users increases, the proposed scheme can access more DUEs to the system compared with conventional scheme because it does not allow users to access who may cause severe interference to others.

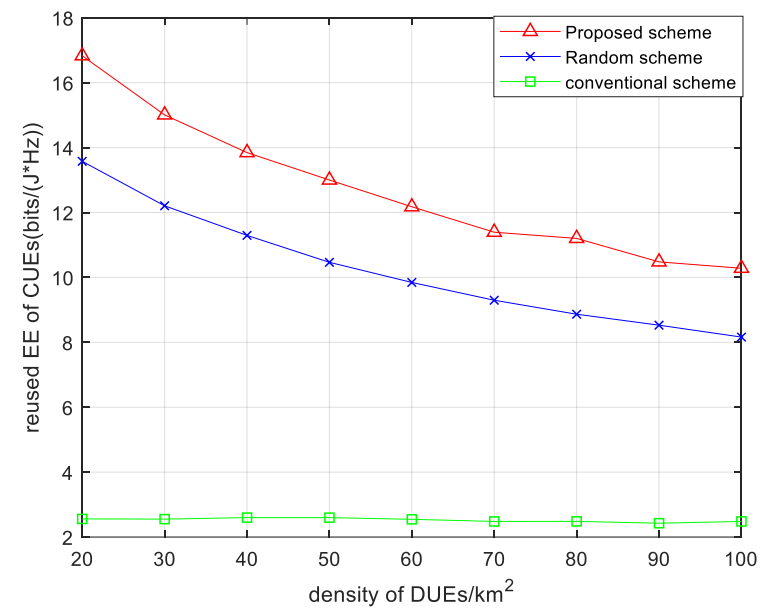

Fig. 5. Energy efficient of CUEs vs Different density of DUEs

As we can see in Fig. 5, compared with conventional scheme, the proposed scheme can avoid severe interference to the CUEs. The reason is that we formulate an ILA for CUE to prevent it suffering from serious interference. And the proposed scheme performs well compared to the random scheme because it carried out a graph coloring algorithm to coordination the interference between users. 


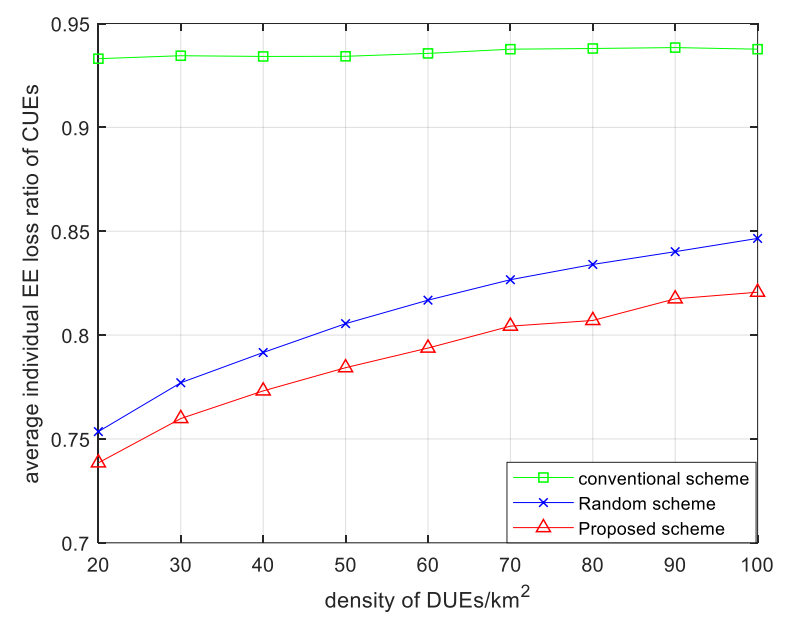

Fig. 6. Average EE loss ratio of CUEs vs Different density of DUEs

Fig. 6 displays the energy efficient loss ratio of CUEs with the different density of DUEs. It can be seen that the EE loss ratio of CUEs under the proposed scheme and random scheme is lower than the conventional scheme due to only CUEs with good channel conditions share spectrum to DUEs in our proposed scheme.

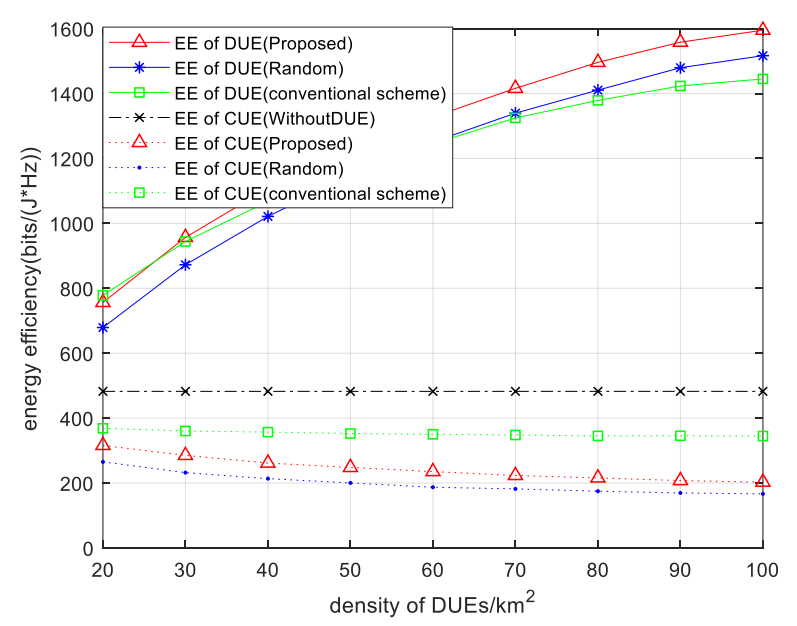

Fig. 7. Energy efficient of users vs Different density of DUEs

The energy efficiency of CUEs and DUEs are illustrated in Fig. 7. As observed, the EE of DUEs increases with the increasing of DUEs' density because the system can access more D2D pairs. And the EE of CUEs decreases with the increasing of DUEs' density. For the EE of DUEs, our proposed scheme performs well than the conventional scheme because it allows more DUEs to access, and it performs well than the random scheme due to it handles the interference problem using the theory of graph-coloring. For the EE of CUEs, contrast with the original system without DUEs, the proposed scheme decreases a bit more than the conventional scheme because the resource of the CUE who has better channel condition is 
reused in the proposed scheme, and the proposed scheme avoids the resource of CUEs with worse channel condition from being reused.

\section{Conclusion}

In this paper, we consider an uplink D2D underlay scenario where the number of DUEs is larger than that of CUEs. In order to improve the access succeed rate of DUEs, we formulate a resource reuse scheme where the available spectrum resource of a CUE is allowed to be reused by multiple DUEs. To maximize the energy efficient under the restrictions, we drive the guard region for CUEs based on random point process to ensure its coverage probability. On the other hand, the performance of DUE can be improved by the ILA which avoids two DUE pairs with small distances sharing the same resource. Furthermore, we proposed an optimal reuse scheme according to the weighted graph coloring theory. Simulation results show that the proposed scheme can significantly improve the system energy efficient and avoid CUEs suffering from serious co-channel interference.

\section{Appendix}

In this Appendix, we will give the proof of Theorem 1.

Proof: The coverage probability can be described as

$$
\begin{aligned}
& \mathrm{P}\left(\frac{P_{C} h_{i} d_{C}^{-\alpha}}{N_{0}+P_{C} h_{0} R_{0}^{-\theta}+\sum_{j \in \Phi \backslash A_{0}} P_{d} h_{j} d_{j}^{-\theta}}>\mathrm{T}\right) \\
&= E\left(\exp \left(-\frac{\mu T d_{C}^{\alpha}}{P_{C}}\left(N_{0}+P_{d} h_{0} R_{0}^{-\theta}+\sum_{j \in \Phi \backslash A_{0}} P_{d} h_{j} d_{j}^{-\theta}\right)\right)\right) \\
&= \frac{\exp \left(-\frac{\mu T d_{C}^{\alpha} N_{0}}{P_{C}}\right)}{1+\frac{T d_{C}^{\alpha} P_{d}^{R_{0}^{-\theta}}}{P_{C}}} E_{\Phi}\left(\prod_{j \in \Phi \backslash A_{0}} \frac{1}{1+\frac{T d_{C}^{\alpha}}{P_{C}} P_{d} d_{j}^{-\theta}}\right) \\
&= \frac{\exp \left(-\frac{\mu T d_{C}^{\alpha} N_{0}}{P_{C}}\right)}{1+\frac{T d_{C}^{\alpha} P_{d} R_{0}^{-\theta}}{P_{C}}} \exp \left(-2 \frac{\pi \lambda_{d}}{M} \int_{R_{0}}^{R_{C}}\left(1-\frac{1}{1+\frac{T d_{C}^{\alpha}}{P_{C}} P_{d} d_{j}^{-\theta}}\right) x d x\right) \\
&= \frac{\exp \left(-\frac{\mu T d_{C}^{\alpha} N_{0}}{P_{C}}\right)}{1+\frac{T d_{C}^{\alpha} P_{d} R_{0}^{-\theta}}{P_{C}}} \exp \left(-2 \frac{\pi \lambda_{d}}{M} \int_{R_{0}}^{R R_{C}}\left(\frac{1}{1+\frac{P_{C} x^{\theta}}{T d_{C}^{\alpha} P_{d}}}\right) x d x\right) \\
&= \frac{\exp \left(-\frac{\mu T d_{C}^{\alpha} N_{0}}{P_{C}}\right)}{1+\frac{T d_{C}^{\alpha} P_{d} R_{0}^{-\theta}}{P_{C}}} \exp \left(-\frac{\pi \lambda_{d}}{M}\left(\frac{T P_{d} d_{C}^{\alpha}}{P_{C}}\right)^{\frac{2}{\theta}} \int^{R_{C}} R_{0}^{2}\left(\frac{P_{C}}{T P_{d} d_{C}^{\alpha}}\right)^{\frac{2}{\theta}}\left(\frac{1}{1+u^{\frac{\theta}{2}}}\right) d u\right) \\
& \triangleq f\left(R_{0}\right)
\end{aligned}
$$

where (a) follows from Lemma 1. (b) follows from the independence of the small-scale fading powers. (c) follows from the probability generating functional [5] of the PPP. The integration limits are from $R_{0}$ to $\infty$ since the closest interferer is at least at a distance $R_{0}$. (d) follows from a change of variables $v=\left(x\left(\frac{P_{c}}{T P_{d} d_{c}^{\alpha}}\right)^{1 / \theta}\right)^{2}$. Notice that $f\left(R_{0}\right)$ is a monotonically increasing function. From (8) and (17), we obtain (10). 


\section{Reference}

[1] R. I. Ansari, C. Chrysostomou, S. A. Hassan, M. Guizani S. Mumtaz, J. Rodriguez, and J. Rodrigues, "5G D2D Networks: techniques, challenges, and future prospects," IEEE Systems Journal, vol. 12, no. 4, pp. 3970-3984, 2018. Article (CrossRef Link)

[2] L. Wei, R. Q. Hu, Y. Qian, and G. Wu, "Enable device-to-device communications underlaying cellular networks: challenges and research aspects," IEEE Communications Magazine, vol. 52, no. 6, pp. 90-96, 2014. Article (CrossRef Link)

[3] P. Jänis, C. Yu, K. Doppler, C. Ribeiro, C. Wijting, K. Hugl, O. Tirkkonen, and V. Koivunen, "Device-to-device communication underlaying cellular communications systems," International Journal of Communications, Network \& System Sciences, vol. 2, no. 3, pp. 169-178, 2009. Article (CrossRef Link)

[4] X. Ge, B. Yang, J. Ye, G. Mao, C. X. Wang, and T. Han, "Spatial spectrum and energy efficiency of random cellular networks," IEEE Transactions on Communications, vol. 63, no. 3, pp. 10191030, 2015. Article (CrossRef Link)

[5] A. Asadi, Q. Wang, and V. Mancuso, "A survey on device-to-device communication in cellular networks," IEEE Communication Surveys \& Tutorials, vol. 16, no. 4, pp. 1801-1819, 2014. Article (CrossRef Link)

[6] M. Zeng, Y. Luo, and H. Jiang, "Energy efficient resource allocation for wireless power transfersupported D2D communication with battery," IEEE Access, vol. 7, pp. 185666-185676, 2019. Article (CrossRef Link)

[7] H. A. Mustafa, M. Z. Shakir, M. A. Imran, A. Imran, and R. Tafazolli, "Coverage gain and deviceto-device user density: stochastic geometry modeling and analysis," IEEE Communications Letters, vol. 19, no. 10, pp. 1742-1745, 2015. Article (CrossRef Link)

[8] A. Abdallah, M. M. Mansour, and A. Chehab, "A distance-based power control scheme for D2D communications using stochastic geometry," in Proc. of vehicular technology conference, pp. 1-6, 2017.

[9] Y. Jiang, Q. Liu, F. Zheng, X. Gao, and X. You, "Energy-efficient joint resource allocation and power control for D2D communications," IEEE Transactions on Vehicular Technology, vol. 65, no. 8, pp. 6119-6127, 2016. Article (CrossRef Link)

[10] X. Gao, H. Han, K. Yang, J. An, and X. Bu, "Fairness-aware energy-efficient power control scheme for D2D communications underlaying cellular networks," in Proc. of IEEE 18th International Workshop on Signal Processing Advances in Wireless Communications, pp. 1-5, 2017. Article (CrossRef Link)

[11] K. Yang, S. Martin, C. Xing, J. Wu, and R. Fan, "Energy-efficient power control for device-todevice communications," IEEE Journal on Selected Areas in Communications, vol. 34, no. 12, pp. 3208-3220, 2016. Article (CrossRef Link)

[12] T. D. Hoang, L. B. Le, and T. Le-Ngoc, "Energy-efficient resource allocation for D2D communications in cellular networks," IEEE Transactions on vehicular technology, vol. 65, no. 9, pp. 6972-6986, 2016. Article (CrossRef Link)

[13] F. Wang, C. Xu, L. Song, and Z. Han, "Energy-efficient resource allocation for device-to-device underlay communication," IEEE Transactions on Wireless Communications, vol. 14, no. 4, pp. 2082-2092, Apr. 2015. Article (CrossRef Link)

[14] T. D. Hoang, L. B. Le, and T. Le-Ngoc, "Energy-efficient resource allocation for D2D communications in cellular networks," Wireless Communications Symposium, pp. 2251-2256, 2015. Article (CrossRef Link)

[15] S. Wen, X. Zhu, Z. Lin, X. Zhang, and D. Yang, "Energy efficient power allocation schemes for device-to-device(D2D) communication," in Proc. of IEEE 78th Vehicular Technology Conference (VTC Fall), pp. 1-5, Sep. 2013. Article (CrossRef Link) 
[16] S. Xu, H. Zhang, J. Tian, S. Guo, and Z. Zhou, "Distributed energy-efficient resource allocation and power control for device-to-device communications underlaying cellular networks," International Symposium on Wireless Personal Multimedia Communications, 2017. Article (CrossRef Link)

[17] H. Min, J. Lee, S. Park, and D. Hong, "Capacity enhancement using an interference limited area for device-to-device uplink underlaying cellular networks," IEEE Transactions on Wireless Communications, vol. 10, no. 12, pp. 3995-4000, Dec. 2011. Article (CrossRef Link)

[18] H. Wang and X. Chu, "Distance-constrained resource-sharing criteria for device-to-device communications underlaying cellular networks," Electronics Letters, vol. 48, no. 9, pp. 528-530, Apr. 2012. Article (CrossRef Link)

[19] F. Xu and P. Pan, "Dynamic frequency reuse based on guard regions in two-layer heterogeneous networks," Electronics Letters, vol. 51, no. 12, pp. 947-949, 2015. Article (CrossRef Link)

[20] X. Zhu, L. Dai, and Z. Wang, "Graph coloring based pilot allocation to mitigate pilot contamination for multi-cell massive MIMO systems," IEEE Communications Letters, vol. 19, no. 10, pp. 18421845, Oct. 2015. Article (CrossRef Link)

[21] J. Huang, C. Xing, and M. Guizani, "Power Allocation for D2D Communications with SWIPT," IEEE Transactions on Wireless Communications, vol. 19, no. 4, pp. 2308-2320, Apr. 2020. Article (CrossRef Link)

[22] J. Huang, C. Huang, C. Xing, Z. Chang, Y. Zhao, and Q. Zhao, "An Energy-Efficient Communication Scheme for Collaborative Mobile Clouds in Content Sharing: Design and Optimization," IEEE Transactions on Industrial Informatics, vol. 15, no. 10, pp. 5700-5707, Oct. 2019. Article (CrossRefLink)

[23] J. Huang, C. Xing, Y. Qian, and Z. Haas, "Resource Allocation for Multi-cell Device-to-Device Communications Underlaying 5G Networks: A Game-Theoretic Mechanism with Incomplete Information," IEEE Transactions on Vehicular Technology, vol. 67, no. 3, pp. 2557-2570, Oct. 2018. Article (CrossRef Link)

[24] J. Huang, Y. Yin, Y. Zhao, Q. Duan, W. Wang, and S. Yu, "A Game-Theoretic Resource Allocation Approach for Intercell Device-to-Device Communications in Cellular Networks," IEEE Transactions on Emerging Topics in Computing, vol. 4, no. 4, pp.475-486, 2016. Article (CrossRef Link)

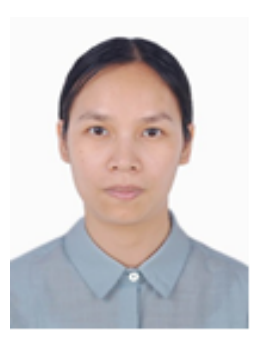

Fangmin Xu received the M.S. degree in Probability and Mathematical Statistics in Beijing University of Technology, China,in 2005, and the Ph.D. degree in communication engineering from Beijing University of Posts and Telecommunications (BUPT). From 2018 to 2019, she was a Visiting Scholar with the Department of Electrical and Computer Engineering, University of Massachusetts, Dartmouth, USA. Currently, she is a faculty member at Hangzhou Dianzi University, in Hangzhou, China. Her research interests include resource allocation in wireless communications, fractional frequency reuse, massive MIMO systems, and wireless network resource allocations.

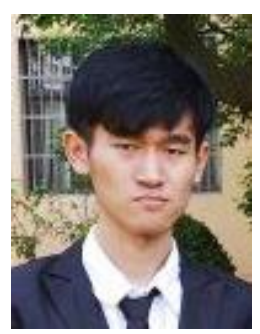

Pengkai Zou received the B.Eng degree in communication engineering from Qingdao University, Qingdao, China, in 2018, and is currently pursuing the M.E. degree in electronics and communication engineering in Hangzhou Dianzi University, Hangzhou.His research interests include heterogeneous networks, stochastic geometry, resource allocations of cellular networks, and next generation wireless communication. 


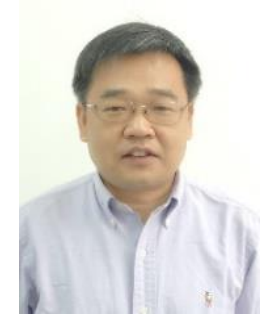

Haiquan Wang received the M.S. degree in Nankai University, China, in 1989, and the Ph.D. degree in Kyoto University, Japan, in 1997, both in mathematics, and the Ph.D. degree in University of Delaware, Newark, in 2005, in electrical engineering. From 1997 to 1998 , he was a Postdoctoral Researcher with the Department of Mathematics, Kyoto University. From 1998 to 2001, he was a Lecturer (part-time) with Ritsumei University, Japan. From 2001 to 2002, he was a Visiting Scholar with the Department of Electrical and Computer Engineering, University of Delaware, Newark. From 2005 to 2008, he was a Postdoctoral Researcher with the Department of Electrical and Computer Engineering, University of Waterloo, Canada. He joined the College of Communications Engineering, Hangzhou Dianzi University, Hangzhou, China, in July 2008 as a faculty member. His research interests include wireless communications, digital signal processing, and information theory.

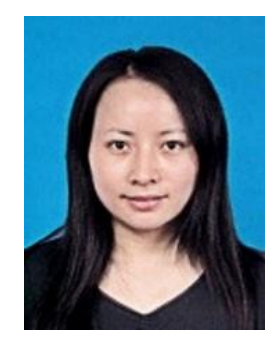

Haiyan Cao received the B.Eng. degree and the M.Eng.degree in communication and information system from Anhui University of Technology, Maanshan, China, in 2000 and 2003, respectively, the the Ph.D. degree in communication and information systems from South China University of Technology, Guangzhou, China, in 2006. From 2015 to 2016, she was a Research Scholar with the Department of Electrical and Computer Engineering, University of Massachusetts Dartmouth,USA. She is currently an Associate Professor with the College of Telecommunication Engineering, Hangzhou Dianzi University, Hangzhou, China. Her research interests include cooperative communications and convex optimization in signal processing and communications.

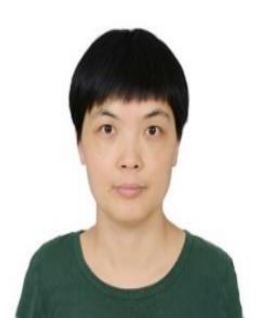

Xin Fang received the B.S. degree in Industrial electrical automation and M.S. degree in Control Theory and Control Engineering, respectively, in Hohai University, Nanjing, China. She received her Ph.D degree in Communication and Information System in Southeast University, Nanjing, China. From September 2004 through March 2006, she was a system engineer in Kaiming Information Co., Ltd. In Shanghai, China. She is currently an Associate Professor in School of Communication Engineering in Hangzhou Dianzi University, in Hangzhou, China. Her research interests span signal processing for wireless communications, massive MIMO systems, and machine learning algorithms for communications.

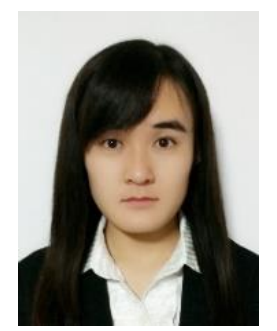

Zhirui Hu received the B.S. degree in communication and information systems from Xi' an University of Posts and Telecommunications, Xi' an, China, in 2010, and the Ph.D. degree in communication and information systems from Beijing University of Posts and Telecommunications, Beijing, China, in 2015. She is currently a lecturer with the College of Telecommunication Engineering, Hangzhou Dianzi University, Hangzhou, China. Her research interests include SWIPT technology, signal processing technology in wireless communication systems. 